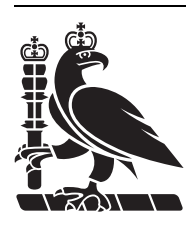

Royal College

\title{
PAEDIATRIC SURGERY
}

\section{Congenital mesoblastic nephroma: a single-centre series}

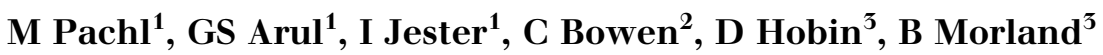

${ }^{1}$ Department of Paediatric Surgery and Urology, Birmingham Children's Hospital, Birmingham, UK ${ }^{2}$ Department of Histopathology, Birmingham Children's Hospital, Birmingham, UK

${ }^{3}$ Oncology Department, Birmingham Children's Hospital, Birmingham, UK

ABSTRACT

BACKGROUND Congenital mesoblastic nephroma is a rare disease. Treatment is surgical in the first instance. Chemotherapy has traditionally been thought not to have a role. Recent literature suggests a $50 \%$ mortality rate for recurrent/metastatic disease.

MATERIALS AND METHODS This study is a retrospective case review of prospectively collected data. Demographics, histopathology, treatment, outcomes and follow up were reviewed.

RESULTS Nine patients, 6 male and 3 female, were included. The median age at presentation was one month (range 0-7 months); follow-up was for a median of 21.5 months (range 16-79 months). Two patients had mixed and classical subtypes and the other five had the cellular subtype. Surgery was completed by an open procedure in eight patients and laparoscopically in one. There were three recurrences; two were local and one was pulmonary. Recurrences were treated with a combination of chemotherapy, radiotherapy and surgery. One patient with recurrent disease died from acute-on-chronic respiratory failure secondary to lung irradiation but was disease free. The other eight are disease free, alive and well with no sequelae at latest follow-up.

CONCLUSIONS Surgery remains the mainstay of management with chemo- and radiotherapy reserved for unresectable tumours or adjuvant management of recurrent disease. Specimen-positive margins are not an indication for instituting chemotherapy. The tyrosine kinase pathway seems to be a potential target for future chemotherapeutic agents although it is too early to assess how that will impact on the management of congenital mesoblastic nephroma.
\end{abstract}

KEYWORDS

Surgery - Chemotherapy - Radiotherapy - Mesoblastic nephroma

Accepted 30 June 2019

CORRESPONDENCE TO

Max Pachl, E: Max.pachl@nhs.net

\section{Introduction}

Congenital mesoblastic nephroma is the most common renal neoplasm in newborns and in early infancy. Of patients presented in a series of 50 cases, $74 \%$ of the patients were younger than four months of age. ${ }^{1}$ Traditional thinking is that congenital mesoblastic nephroma is a surgically cured benign disease and neoadjuvant or adjuvant therapy has little to offer.

The tumour was first described by Bolande et al in 1967 as a renal tumour of infancy and is subdivided histologically into classical, mixed and cellular subtypes. ${ }^{2}$ The classical subtype is thought to be benign, with recurrent or metastatic disease occurring in the mixed and cellular subtypes.

Genetic crossover is seen with congenital infantile fibrosarcoma as both can have a $\mathrm{t}(12 ; 15)(\mathrm{p} 13 ; \mathrm{q} 25)$ translocation resulting in a chimeric protein tyrosine kinase ETV6-
NTRK3 fusion gene product. ${ }^{3}$ However, the inference of this with regard to $\mathrm{CMN}$ is unclear.

A recent review paper has suggested that the mortality in recurrent and/or metastatic congenital mesoblastic nephroma is $50 \%$ and that there was no difference in outcome among the patients who received chemotherapy compared with those who were treated by surgery alone. ${ }^{4}$

We wanted to review our series to add to the body of medical evidence and to propose that options for adjuvant chemotherapy in unresectable recurrent disease can obtain long-term complete remission.

\section{Materials and methods}

This study was a retrospective case note review of prospectively collected data. Patients were identified from the West Midlands Regional Children's Tumour Registry at Birmingham Children's Hospital. Patients who presented to 
Birmingham Children's Hospital between January 2008 and November 2017 and were subsequently diagnosed with congenital mesoblastic nephroma were included.

Patient demographics, tumour biology, histopathology and surgical details were reviewed. Evidence of recurrence or metastases were included along with any neoadjuvant or adjuvant chemo- or radiotherapy. Details of follow-up and disease status were also documented. Congenital mesoblastic nephromas were histologically classified as classical, mixed or cellular based on accepted criteria, ${ }^{5,6}$ and were staged according to the International Society of Pediatric Oncology (SIOP) staging criteria. ${ }^{7}$

\section{Results}

Nine patients were identified, of whom six were male and three female. Age at presentation was a median of one month (range antenatal to seven months). Follow-up was a median of 21.5 months (range 16-79 months; Table 1) with ultrasound performed three-monthly in asymptomatic cases and magnetic resonance imaging (MRI) in other cases. Mixed and classical subtypes each occurred in two patients and the other five had the cellular subtype. The presence of ETV6-NTRK3 fusion gene rearrangement was tested for in seven patients and was present in two. All patients underwent radical nephroureterectomy, eight by the open technique and one laparoscopically. Only three patients had a complete microscopic excision; five had positive microscopic margins and one had positive macroscopic margins noted at surgery.

Three of our nine patients went on to have recurrent disease (Table 2), of which two were local recurrences and one was pulmonary. Of these, two had the cellular subtype and one the mixed subtype. One of these was a SIOP stage 1 disease following primary nephrectomy (patient 5). All three of these patients received chemotherapy.

The local recurrence in patient 6 occurred in the setting of residual macroscopic disease following resection for a life-threatening massive intra-abdominal tumour. This patient had received two cycles of neoadjuvant vincristine and actinomycin chemotherapy (VA) because the lesion was initially thought to be unresectable, but her clinical condition deteriorated and she underwent an attempted full resection. At surgery, macroscopic disease was noted to remain on the pancreas and the retrohepatic vena cava. Following resection, the tumour recurred after two months. The patient was treated with 12 months of vincristine, adriamycin and cyclophosphamide (VAC) chemotherapy, in line with the UK high-risk Wilms tumour protocol. She underwent repeated MRI, which showed gradual resolution of the recurrent lesion and she attained complete remission on further scans.

The other local recurrence occurred in patient 2 who had microscopic residual disease at the surgical margins following resection of a mixed congenital mesoblastic nephroma. The tumour recurred five months after her initial surgery and was unresectable at relapse. She had four cycles of VA chemotherapy and then underwent a further resection of residual disease. On this occasion the surgical margins were again microscopically positive for disease. She received a further six cycles of cyclophosphamide and doxorubicin and subsequently attained complete remission based on MRI.

Patient 5 had a complete resection of a cellular congenital mesoblastic nephroma and was noted to have a thoracic recurrence two months following primary surgery. She received two cycles of VAC and subsequently had progressive disease on imaging. Chemotherapy was changed to vincristine, doxorubicin, cyclophosphamide (VDC) and a middle lobectomy was performed seven months after primary resection. This confirmed the presence of metastatic congenital mesoblastic nephroma, which was completely excised. The patient then received lung radiotherapy. The combination of lobe resection and radiotherapy resulted in reduced lung capacity and fibrosis. The patient ultimately succumbed due to a combination of recurrent chest

\begin{tabular}{|c|c|c|c|c|c|c|c|c|c|}
\hline $\begin{array}{l}\text { Patient } \\
\text { number }\end{array}$ & $\begin{array}{l}\text { Age at } \\
\text { presentation } \\
\text { (months) }\end{array}$ & $\begin{array}{l}\text { Male/ } \\
\text { female }\end{array}$ & $\begin{array}{l}\text { Histological } \\
\text { subtype }\end{array}$ & Stage & $\begin{array}{l}\text { Method of } \\
\text { excision }\end{array}$ & Resection margins & ETV6 & Recurrence & $\begin{array}{l}\text { Follow up } \\
\text { (months) }\end{array}$ \\
\hline 1 & 0 & M & Mixed & 2 & Open & Negative & Negative & No & 21 \\
\hline 2 & 3 & $\mathrm{~F}$ & Mixed & 3 & Open & Microscopic positive & Negative & Yes & 61 \\
\hline 3 & 1 & M & Classical & 3 & Open & Microscopic positive & Negative & No & 19 \\
\hline 4 & 1 & M & Cellular & 3 & Open & Microscopic positive & Positive & No & 73 \\
\hline 5 & 7 & $\mathrm{~F}$ & Cellular & 2 & Open & Negative & Not tested & Yes & $\mathrm{N} / \mathrm{A}$ \\
\hline 6 & 2 & $\mathrm{~F}$ & Cellular & 3 & Open & Macroscopic positive & Not tested & Yes & 16 \\
\hline 7 & 0 & $\mathrm{M}$ & Classical & 3 & Open & Microscopic positive & Negative & No & 19 \\
\hline 8 & 1 & M & Cellular & 1 & Laparoscopic & Negative & Negative & No & 22 \\
\hline 9 & 0 & M & Cellular & 3 & Open & Microscopic positive & Positive & No & 79 \\
\hline
\end{tabular}




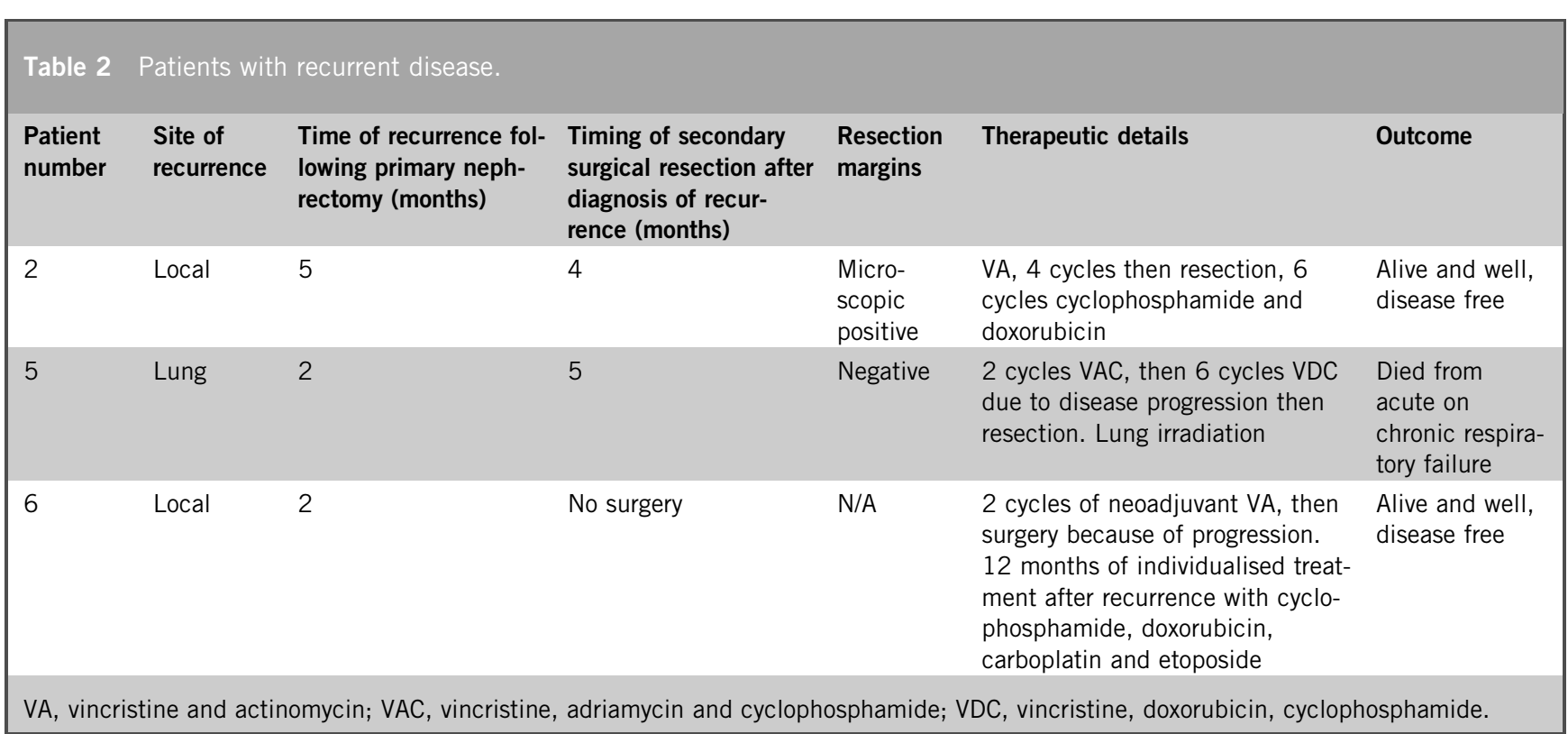

infections and lung fibrosis 15 months following resection of the primary disease. She was in complete remission prior to her death.

\section{Discussion}

Congenital mesoblastic nephroma is a rare tumour occurring in one in 25,000 and so making conclusions from the medical evidence is difficult. ${ }^{8}$ Scattered case reports abound of tumours treated by different methods and there are likely to be a significant number of patients lost to the medical literature.

The histopathology of congenital mesoblastic nephroma is well documented. ${ }^{5,9}$ The classical type is the most benign, with no cases of recurrence or metastasis reported. This is in contrast to the mixed and cellular types, with the cellular type showing the most aggressive nature. Most patients with cellular mesoblastic nephromas do extremely well and relapse occurs only in rare cases. The vast majority of cases are treated with surgery alone, as in our series.

In their review paper, Gooskens et al showed that the risk of relapse is highest in stage 3 disease, at $23 \%$, and that all these patients had a cellular subtype. ${ }^{10}$ As shown above, the local recurrences in our patients occurred in those who had residual disease at surgical margins. However this does not mean that positive margins mandate recurrent disease as there are four other patients in this series who had positive margins who did not have recurrent disease. Two of those had the classical subtype and other two the cellular subtype. Conversely, the patient with lung recurrence had a complete resection with negative margins at primary nephrectomy. It is possible that the lung recurrence was an initial microscopic metastasis that was invisible to the imaging, but there are other reports in the literature documenting pulmonary recurrent disease. ${ }^{11,12}$
There seemed to be no correlation between ETV6NTRK3 positivity and outcomes in our small series, although current research in this area is promising. There is one report of an ETV6-NTRK3-positive congenital mesoblastic nephroma showing regression with chemotherapy prior to primary nephrectomy but planned early surgery remains the mainstay of treatment. ${ }^{13}$ In addition to an ETV6-NTRK3, an EML4-NTRK3 gene fusion product has recently been described in relation to infantile fibrosarcoma and congenital mesoblastic nephroma, showing that there is an increasing number of gene fusion products linking these two historically different tumours. ${ }^{14}$

El Demallawy et $a l,{ }^{15}$ in an elegant histopathological study, took this association a step further and suggested that the absence of expression of stem-cell markers and markers involved in urogenital development implied that congenital mesoblastic nephroma is not a nephroma at all and most likely represents a soft-tissue tumour. They also surmised that congenital infantile fibrosarcoma represented a cellular congenital mesoblastic nephroma with a predilection to arise in the kidney.

Numerous other studies in both adult and paediatric tumours show a link between NTRK gene fusion products and congenital mesoblastic nephroma, together with other tumours including glioblastoma,${ }^{16}$ gastrointestinal stromal tumours ${ }^{17}$ and acute myeloid leukaemia. ${ }^{18}$ There is hope that a targeted therapy for NTRK-positive congenital mesoblastic nephroma and infantile fibrosarcoma could be useful in the neoadjuvant or adjuvant treatment of recurrent and/or metastatic congenital mesoblastic nephroma. The tropomyosin receptor kinase pathway is the lead candidate, for which therapeutic targets already exist and are under investigation. ${ }^{19}$

For the one patient who had a tumour that was initially deemed unresectable, neoadjuvant chemotherapy had no initial effect. However, surgery was required on an 
emergency basis because of the patient's deteriorating respiratory condition. We do not know whether it had been possible to administer chemotherapy over a longer period of time or whether there may have been some reduction in tumour volume. England et al reported one patient who received preoperative chemotherapy due to the large tumour size, with a dramatic reduction in tumour volume. ${ }^{8}$ There may therefore be some benefit in giving neoadjuvant chemotherapy in selected cases; for example, where primary resection is deemed impossible or unsafe. The value of lung radiotherapy in lung metastases/recurrence is of unknown benefit and in our patient it seems to have contributed to her acute-on-chronic fatal lung disease process.

In our cohort, patient 8 underwent a laparoscopic tumour nephroureterectomy of a mesoblastic nephroma measuring $5 \times 5 \times 5 \mathrm{~cm}$ by the corresponding author (MP) at six weeks of age and a weight of $4 \mathrm{~kg}$. Surgery gave a complete resection with negative margins, proving that this approach is feasible even in the neonatal population and gives an equivalent oncological outcome to open surgery in highly selected patients. It also gives a better cosmetic result along with a reduced length of stay. We would recommend that this technique is only attempted by paediatric oncology surgeons who have significant expertise in minimal access surgery.

There are clear limitations to our series, namely that this is a single-centre series over only nine years. However the data produced are important to share with other centres dealing with the management of these sometimes difficult and complex tumours.

In our series there were no deaths due to disease process in any patient including the three with recurrent and/ or metastatic tumours. This is contrast to that described in the recent paper by Jehangir et al, which documented a $50 \%$ mortality rate from recurrent and/or metastatic congenital mesoblastic nephroma. ${ }^{4}$

\section{Conclusions}

In summary, congenital mesoblastic nephroma remains a highly curable disease and surgery at presentation and for the management of recurrent disease remains the mainstay of treatment. A positive microscopic/macroscopic tumour margin is not an indication to start chemotherapy, which should be reserved for recurrent, unresectable tumours. However, a second surgical excision should be regarded as the mainstay of therapy, even in the relapse setting. The choice of chemotherapy is challenging based on the relatively few reported cases, but a 'Wilms'-style' approach is not unreasonable to consider with vincristine, dactinomycin and consideration of the addition of anthracyclines and alkylating drugs depending on the patient's response. Radiotherapy can be considered but its short-, medium- and long-term effects should be carefully evaluated on an individual basis.

\section{References}

1. Furtwaengler R, Reinhard H, Leuschner I et al. Gesellschaft fur Pädiatrische Onkologie und Hämatologie (GPOH) Nephroblastoma Study Group. Mesoblastic nephroma: a report from the Gesellschaft fur Pädiatrische Onkologie und Hämatologie (GPOH). Cancer 2006; 106: 2,275-2,283.

2. Bolande RP, Borough AJ, Izant RJ Jr. Congenital mesoblastic nephroma of infancy: a report of eight cases and the relationship to Wilms' tumor. Paediatrics 1967; 40: 272-278.

3. Sandberg AA, Bridge JA. Updates on the cytogenetics and molecular genetics of bone and soft tissue tumors: congenital (infantile) fibrosarcoma and mesoblastic nephroma. Cancer Genet Cytogenet 2002; 132: 1-13.

4. Jehangir S, Kurian JJ, Selvarajah D et al. Recurrent and metastatic congenital mesoblastic nephroma: where does the evidence stand? Pediatr Surg Int 2017; 33: $1,183-1,188$.

5. Sandstedt B, Delemarre J, Krul EJ et al. Mesoblastic nephromas: a study of 29 tumours from the SIOP nephroblastoma file. Histopathology 1985; 9: 741-750.

6. Beckwith B,Weeks D. CMN when should we worry? Arch Pathol Lab Med 1986; 110: 98-99.

7. Vujanic GM, Sandstedt B, Harms D et al. Revised International Society of Paediatric Oncology (SIOP) working classification of renal tumors of childhood. Med Pediatr Oncol 2002; 38: 79-82.

8. England RJ, Haider N, Vujanic GM et al. Mesoblastic nephroma: a report of the United Kingdom children's cancer and leukaemia group (CCLG). Pediatr Blood Cancer 2011; 56(5): 744-748.

9. Argani P, Ladanyi M. Recent advances in pediatric renal neoplasia. Adv Anat Pathol 2003; 10(5): 243-260.

10. Gooskens SL, Houwing ME, Vujanic GM et al. Congenital mesoblastic nephroma 50 years after its recognition: a narrative review. Pediatr Blood Cancer 2017; 64: e26437.

11. Argani P, Fritsch M, Kadkol SS et al. Detection of the ETV6-NTRK3 chimeric RNA of infantile fibrosarcoma/cellular congenital mesoblastic nephroma in paraffin-embedded tissue: application to challenging pediatric renal stromal tumors. Mod Pathol 2000; 13: 29-36.

12. Vujanic GM, Delemarre JF, Moeslichan S et al. Mesoblastic nephroma metastatic to the lungs and heart: another face of this peculiar lesion: case report and review of the literature. Pediatr Pathol 1993; 13: $143-153$.

13. Whittle S, Gosain A, Scott Brown PY et al. Regression of a Congenital Mesoblastic Nephroma. Pediatr Blood Cancer 2010; 55: 364-368.

14. Church AJ, Calicchio ML, Nardi V et al. Recurrent EML4-NTRK3 fusions in infantile fibrosarcoma and congenital mesoblastic nephroma suggest a revised testing strategy. Mod Pathol 2018; 31(3): 463-473.

15. El Demellawy D, Cundiff CA, Nasr A et al. Congenital mesoblastic nephroma: a study of 19 cases using immunohistochemistry and ETV6-NTRK3 fusion gene rearrangement. Pathology 2016; 48: 47-50.

16. Wu G, Diaz AK, Paugh BS et al. The genomic landscape of diffuse intrinsic pontine glioma and pediatric non-brainstem high-grade glioma. Nat Genet 2014; 46: 444-450.

17. Brenca M, Rossi S, Polano M et al. Transcriptome sequencing identifies ETV6NTRK3 as a gene fusion involved in GIST. J Pathol 2016; 238: 543-539.

18. Kralik JM, Kranewitter W, Boesmueller $\mathrm{H}$ et al. Characterization of a newly identified ETV6-NTRK3 fusion transcript in acute myeloid leukemia. Diagn Pathol 2011; 6: 19

19. Alessio Amatu, Andrea Sartore-Bianchi, Salvatore Siena. NTRK gene fusions as novel targets of cancer therapy across multiple tumour types. ESMO Open 2016; 1: e000023. 